\title{
Degradation kinetics and structural characteristics of pectin under simultaneous sonochemical-enzymatic functions
}

Xiaobin Ma ${ }^{a, 1}$, Wenjun Wang ${ }^{a, 1}$, Danli Wang ${ }^{a}$, Tian Ding ${ }^{a, c}$, Xingqian Ye ${ }^{a, b, c}$, Donghong Liu ${ }^{a, b, c, *}$

a College of Biosystems Engineering and Food Science, Zhejiang University, Hangzhou 310058, China

${ }^{\mathrm{b}}$ Fuli Institute of Food Science, Zhejiang University, Hangzhou 310058, China

${ }^{\mathrm{c}}$ Zhejiang Key Laboratory for Agro-Food Processing, Zhejiang R\&D Center for Food Technology and Equipment, Hangzhou 310058, China

${ }^{1}$ These authors contributed equally to this article.

\section{* Corresponding author}

Address: College of Biosystems Engineering and Food Science, Zhejiang University, 866 Yuhangtang Rd., Hangzhou 310058, China.

Tel.: +86 057188982169; Fax: +86 057188982144; E-mail: dhliu@ zju.edu.cn 


\section{Abstract}

This study investigated the degradation kinetics and structural properties of pectin with combining ultrasound and pectinase treatment. Ultrasound at an intensity of 4.5 $\mathrm{W} \mathrm{mL} \mathrm{m}^{-1}$ and a time of 10 min significantly enhanced the enzymatic degradation of pectin weight-average molecular weight $\left(M_{w}\right)$. The degradation kinetics model of pectin followed $1 / M w_{t}-1 / M w_{0}=k t$, suggesting the randomness of the degradation process. Synergistic effects of ultrasound and pectinase were observed at 20-60 ${ }^{\circ} \mathrm{C}$ and were more effective at lower temperatures. Furthermore, the degree of methoxylation $(\mathrm{DM})$ of sonoenzymolysis pectin significantly decreased whereas the degree of acetylation (DAc) remained unchanged compared to the original and enzymolysis pectin. Simultaneous functions of ultrasound and pectinase caused severe decomposition in pectin homogalacturonan (HG) regions without altering the monosaccharides types, configurations and glycoside linkages of the pectin samples. The complex polymeric structures of pectin transformed into smaller units with simpler branches and shorter chains after sonoenzymolysis reactions.

Keywords: molecular weight; kinetics model; synergistic effect; demethoxylation; primary structure; nanostructure 


\section{Introduction}

Pectin is an important component of the plants cell walls and is widely applied in the food, pharmacy and cosmetic industries (MAY, 1990). Characterization of pectin is difficult given its large molecular weight and complex structures. The identified compositions of pectin include homogalacturonan (HG), rhamnogalacturonan I (RG-I), rhamnogalacturonan II (RG-II) and xylogalacturonan (XG) (Maxwell, Belshaw, Waldron \& Morris, 2012). The RG-II and XG regions (which are highly branched structures bound at HG regions) are less common in pectin structures. The HG region is the "smooth region" of pectin and mainly consists of 1 , 4- $\alpha$-D-galacturonic acid (GalUA). Carboxyl groups of GalUA are partially methylesterified or acetylated; degrees of methoxylation (DM) and acetylation (DAc) are known to determine many functional properties (such as gelling, stabilizing and emulsifying abilities) of pectin (Lofgren, Guillotin \& Hermansson, 2006). RG-I regions constitute as the "hairy region" and contain the backbone of a repeating disaccharide unit (GalUA and rhamnose (Rha) residues) with neutral sugar side chains binding to the Rha residues. As more and more studies are focused on the structure-function relationships of pectin, the RG-I region has attracted great attention because of its potential anti-cancer activity (Maxwell et al., 2012). A proposed mechanism for this bioactivity is that the specific galactan structures contained in RG-I side chains could restrain galectin-3, a protein whose overexpression is observed in many cancers (Fan et al., 2010; Gunning, Bongaerts \& Morris, 2009). Based on this conclusion, pectin structures are modified to design functional products, which are 
called "modified pectin” (MP).

Generally, production procedures of MP can be divided into two steps: de-polymerization of HG regions and modification of RG-I side chains (Maxwell et al., 2012). As an important process of MP production, degradation of pectin HG regions is commonly conducted by chemical or enzymatic treatments. Despite the high efficiency of these traditional methods, pollution problems and expensive costs restrict their applications. Current studies also utilize ultrasound to degrade pectin (Zhang et al., 2013a; Zhang et al., 2013b; Zhang, Zhang, Liu, Ding \& Ye, 2015). A previous study (Zhang et al., 2013a) found that the weight-average molecular weight $\left(M_{w}\right)$ and DM of apple pectin effectively decreased after sonication. Nevertheless, pectin would not be thoroughly degraded by ultrasound due to the decrement of energy transmission under high-intensity or long-time ultrasound irradiation (Sun, Ma, Ye, Kakuda \& Meng, 2010; Zhang et al., 2013b). In this case, development of a green, efficient and economical method for pectin depolymerization becomes an urgent need.

Recently, there has been growing evidence that the simultaneous function of ultrasound and enzymes is an ideal method for polymer degradation. Effectiveness of this combination method is attributed to the improved enzyme activity under ultrasound irradiation (Prajapat, Subhedar \& Gogate, 2016a). Under mild ultrasound conditions, oscillation and rupture of small-amplitude cavitation bubbles lead to the generation of shear forces and free radicals, which could favorably alter enzyme structures and cause the exposure of more active domains (Zhang, Fu \& Liang, 2008). Also, ultrasound depolymerizes the polymer substrate, which causes the resultant 
dispersed structures to be more conducive to enzyme action (Subhedar \& Gogate, 2013). Furthermore, micro-jets produced by ultrasound cavitation accelerate mass transfer in the reaction system (Warmoeskerken, van der Vlist, Moholkar \& Nierstrasz, 2002). All these factors contribute to the improvement of enzyme activity under a mild ultrasonic field. However, due to the complexity and susceptibility of enzyme structures, fierce mechanical and chemical effects formed from intense ultrasound conditions will inhibit enzyme activity and retard degradation processes (Islam, Zhang \& Adhikari, 2014; Ma et al., 2011; Subhedar \& Gogate, 2014). Therefore, variations in enzyme activity strongly depend on operating conditions. Combination of ultrasound and enzymes has been observed to promote degradation of a series of polymers, such as dextran (Bashari et al., 2013), cellulose (Prajapat, Das \& Gogate, 2016b; Szabo \& Csiszar, 2013), guar gum (Prajapat et al., 2016a), xylan (Sun, Zhang, Xiao \& Jin, 2015), pectin (Ma et al., 2016), etc. Our previous study (Ma et al., 2016) applied the combination of ultrasound and pectinase to pectin degradation. Results indicated that reducing sugar productions were increased by $32.59 \%$ under an ultrasonic field of $4.5 \mathrm{~W} \mathrm{~mL}^{-1}$ intensity for $10 \mathrm{~min}$, compared with the traditional enzymatic hydrolysis.

Although there have been some studies on sonoenzymolysis treatments of polymers, the research focus is mainly on the yield of products rather than changes in product structures. The present work evaluated the effect of the combination method from a perspective of substrate structures variations. Pectin $M_{w}$ was designated as an indicator of the degradation extent. Degradation kinetics of sonolysis, enzymolysis 
and sonoenzymolysis were studied to estimate the synergistic effects of ultrasound and pectinase. Detailed structural information, including the DM, DAc, monosaccharide component, primary structures and nanostructures of the degradation products were investigated in order to elucidate the degradation paths of pectin under simultaneous sonochemical-enzymatic effects and provide a theoretical basis for the practical applications of the combination method in MP preparations.

\section{Materials and Methods}

\subsection{Chemicals}

Pectinase from Aspergillus niger (major component: polygalacturonase), pectin from a citrus peel, standard dextran $\left(M_{w}: 670,270,150,50,25\right.$ and $\left.12 \mathrm{kDa}\right)$ and standard monosaccharides (including mannose (Man), Rha, glucuronic acid, GalUA, lactose, glucose (Glu), galactose (Gal), xylose (Xyl), arabinose (Ara) and fucose (Fuc)) were purchased from Sigma-Aldrich (Shanghai, China). Methyl alcohol, acetonitrile, isopropanol and 1-phenyl-3-methyl-5-pyrazolone (PMP) were of HPLC-grade; other chemicals were of analytical grade. All chemicals were used as received from the supplier without further purification.

\subsection{Degradation of pectin catalyzed by pectinase}

The pectinase and pectin powders were dissolved in $1 \mathrm{~mol} \mathrm{~L}^{-1}$ citric acid-phosphate buffer at a $\mathrm{pH}$ of 4.0 with the ultimate concentrations of $2 \mathrm{mg} \mathrm{mL}^{-1}$ and $5 \mathrm{mg} \mathrm{mL} \mathrm{m}^{-1}$, respectively. One milliliter of pectinase samples was added to nineteen milliliters of pectin solution in conical flasks, which were placed in a shaking water-bath. The enzymolysis reactions were carried out at different temperatures 
ranging from $20{ }^{\circ} \mathrm{C}$ to $60{ }^{\circ} \mathrm{C}$ for $2 \mathrm{~min}$ to $40 \mathrm{~min}$. After incubation, the samples were immediately put in a boiling water-bath at $100{ }^{\circ} \mathrm{C}$ for 3 min to inactivate the enzyme.

\subsection{Ultrasound treatments}

In the sonoenzymolysis reactions, the mixtures were sonicated by a probe ultrasonic processor (JY92-IIDN, Ningbo Scientz Biotechnology Co., Ningbo, China) with a $1 \mathrm{~cm}$ diameter horn micro tip. The instrument was operated at $22 \mathrm{kHz}$ and had a maximum power of $900 \mathrm{~W}$. One milliliter of the enzyme sample and nineteen milliliters of pectin solution were mixed in a cylindrical glass reactor (with an inner diameter of about $2.77 \mathrm{~cm}$ ), and the generator probe was immediately inserted (about $1 \mathrm{~cm}$ below the liquid level) to dissipate ultrasonic energy. The reactor was immersed into a low-temperature thermostatic water bath (DC-1006, Safe Corporation, Ningbo, China) in order to keep the solution temperature at a constant. The mixture was processed at different ultrasound intensities ranging from 0.9 to $9 \mathrm{~W} \mathrm{~mL}^{-1}$ for different times $(5-40 \mathrm{~min})$ at $30{ }^{\circ} \mathrm{C}$. Sonoenzymolysis reactions were also conducted at $20-60{ }^{\circ} \mathrm{C}$ for $2-10$ min to investigate the degradation kinetics of pectin molecular weight at each temperature. After the degradation treatments, the mixture was immediately put in a boiling water-bath at $100{ }^{\circ} \mathrm{C}$ for $3 \mathrm{~min}$ to inactivate the enzyme.

In the sonolysis reaction, $1 \mathrm{~mL}$ buffer was added instead of the prepared pectinase sample. The pectin solution was treated with ultrasound as described above.

\subsection{Determination of molecular weight and its polydispersity}

In this study, the molecular weight was denoted as the weight-average molecular weight $\left(M_{w}\right)$, while its distributions were quantified by calculating the polydispersity 
index $\left(=M_{w} / M_{N}\right)$. The molecular parameters of pectin samples were measured using SEC-HPLC as described by Zhang et al. (Zhang et al., 2013a) with some modifications. The Waters 1525 HPLC system (Waters, US) was equipped with a TSK-GEL mixed-bed column $(\mathrm{G} 4000 \mathrm{PW}$ XL $, 300 \times 7.8 \mathrm{~mm}, 10 \mu \mathrm{m}$; Tosoh Bioscience, Tokyo, Japan). The degradation samples were dialyzed with the dialysis tube MD 34 $\left(M_{w}: 8000-14000 \mathrm{kDa}\right)$ in distilled water for $48 \mathrm{~h}$ and then filtered through a $0.45 \mu \mathrm{m}$ membrane before injection. The prepared pectin solution $(40 \mu \mathrm{L})$ flowed through the system with $0.2 \mathrm{M} \mathrm{NaCl}$ as the mobile phase at $0.5 \mathrm{~mL} / \mathrm{min}$ and $40{ }^{\circ} \mathrm{C}$. After $30 \mathrm{~min}$ of elution, the eluent was monitored with a refractive index detector (Waters 2414, US). The molecular weight and its polydispersity of pectin samples were calculated using Breeze 2 GPC.

\subsection{Degradation kinetics of pectin $M_{w}$ and calculations of synergistic coefficients}

The degradation kinetics model of the pectin molecular weight was investigated by graphical analysis. The random depolymerization reactions were supposed to abide by the second-order kinetics model according to Roubroeks et al. (Roubroeks, Andersson, Mastromauro, Christensen \& Aman, 2001):

$$
1 / M w_{t}-1 / M w_{0}=k t
$$

where $M w_{t}$ and $M w_{0}$ are $M w(\mathrm{kDa})$ of pectin at reaction time $t$ and 0 , respectively; $t$ is the reaction time ( $\mathrm{min})$ and $\mathrm{k}$ is the rate constant $\left(\mathrm{mol} \mathrm{g}^{-1} \mathrm{~min}^{-1}\right)$ of different degradation reactions.

Synergistic coefficients were calculated by the second-order rate constants and 
the following equation:

$$
S=\frac{k_{\text {Sonoenzymolysis }}-\left(k_{\text {sonolysis }}+k_{\text {enzymolysis }}\right)}{k_{\text {sonoenzymolysis }}} \times 100(\%)
$$

where $S$ is the synergistic coefficient $(\%)$, and $k_{\text {sonolysis }}, k_{\text {enzymolysis }}$ and $k_{\text {sonoenzymolysis }}$ are the second-order rate constants $\left(\mathrm{mol} \mathrm{g}^{-1} \mathrm{~min}^{-1}\right)$ of the sonolysis, enzymolysis and sonoenzymolysis reactions, respectively. The effects between ultrasound and pectinase can be judged as follows: $S>0$, synergistic effect; $S=0$, additive effect; $S<0$, antagonistic effect.

\subsection{Determination of pectin DM and DAc}

All the structural identification experiments below were conducted with three samples: original pectin, enzymolysis pectin (hydrolyzed at $30{ }^{\circ} \mathrm{C}$ for $10 \mathrm{~min}$ ) and sonoenzymolysis pectin (degraded at $4.5 \mathrm{~W} \mathrm{~mL}^{-1}$ intensity and $30{ }^{\circ} \mathrm{C}$ for 10 min).Values of DM and DAc of pectin samples were determined by HPLC as described by Levigne et al. (Levigne, Thomas, Ralet, Quemener \& Thibault, 2002) with slight modifications. The freeze-dried pectin $(5 \mathrm{mg})$ was suspended in $1.5 \mathrm{~mL}$ of a solution consisting of $2 \mathrm{mM} \mathrm{CuSO}_{4}, 0.3 \mathrm{M} \mathrm{NaOH}$ and $4 \mathrm{mM}$ isopropanol as an internal standard. The mixture was reacted at $4{ }^{\circ} \mathrm{C}$ for $30 \mathrm{~min}$ and then centrifuged at $5000 \mathrm{r}$ for $10 \mathrm{~min}$. After that, the $\mathrm{pH}$ of the supernatant was adjusted to $2.0 \mathrm{using}$ diluted $\mathrm{HCl}$ solution. Samples $(20 \mu \mathrm{L})$ were injected into the Waters 1525 HPLC system (Waters, US) equipped with a $\mathrm{C}_{18}$ column (SinoChrom ODS-BP, $250 \times 4.6$

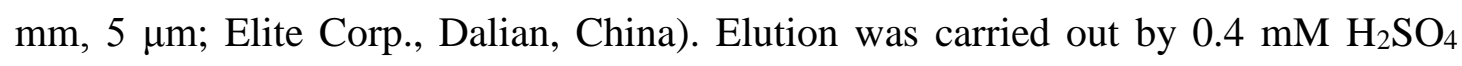
solution and passed through the system at a flow rate of $0.8 \mathrm{~mL} / \mathrm{min}$ at $25{ }^{\circ} \mathrm{C}$, with refractive index detection. Methyl alcohol (23\%, volume fraction), glacial acetic acid 
(4\%) and isopropanol (4\%) were mixed and used as standards.

\subsection{Determination of the monosaccharide composition of pectin samples}

The monosaccharide contents of degradation product was measured using HPLC as described by Zhang et al. (Zhang et al., 2013a) with some modifications.

\subsubsection{Acid hydrolysis of pectin samples and precolumn derivatization}

The freeze-dried product ( $2 \mathrm{mg}$ ) was dissolved into $2 \mathrm{~mL}$ of trifluoroacetic acid (2 M) in an ampoule bottle, and the hydrolysis reaction was conducted at $110{ }^{\circ} \mathrm{C}$ for 8 h. The acid-hydrolyzed product was then dried and neutralized by $\mathrm{NaOH}(0.1 \mathrm{M})$.

$\mathrm{NaOH}$ solution $(0.3 \mathrm{M}, 450 \mu \mathrm{L})$, methanol solution of $\mathrm{PMP}(0.5 \mathrm{M}, 450 \mu \mathrm{L})$ and lactose $(0.02 \mathrm{M}, 50 \mu \mathrm{L})$ as an internal standard were added to the acidolysis samples $(400 \mu \mathrm{L})$. The reagent was reacted at $70{ }^{\circ} \mathrm{C}$ for $30 \mathrm{~min}$ and then neutralized with 450 $\mu \mathrm{L}$ of $\mathrm{HCl}$ solution $(0.3 \mathrm{M})$. Acquired mixtures were extracted by $2 \mathrm{~mL}$ of chloroform and the operation was repeated twice. The aqueous layer was collected and filtered with a $0.45 \mu \mathrm{m}$ membrane prior to HPLC analysis.

\subsubsection{HPLC measurement}

HPLC measurement was conducted in a Waters 2695 HPLC system (Waters, US) equipped with a $\mathrm{C}_{18}$ column (Zorbax Aclips $\mathrm{XDB}, 250 \times 4.6 \mathrm{~mm}, 5 \mu \mathrm{m}$; Agilent Technologies Inc., CA, USA) and a PDA 2996 detector (Waters, US).

Acetonitrile was dissolved in the $\mathrm{KH}_{2} \mathrm{PO}_{4}-\mathrm{NaOH}$ buffer $(0.05 \mathrm{M}, \mathrm{pH}$ 6.9) to obtain the mobile phase A (with acetonitrile contents of 15\%) and B (with acetonitrile contents of 40\%). During HPLC analysis, a gradient time pattern was applied with the following times: $0 \mathrm{~min} \rightarrow 10 \mathrm{~min} \rightarrow 30 \mathrm{~min} \rightarrow 35 \mathrm{~min} \rightarrow 40 \mathrm{~min}$; and the corresponding 
concentration gradient of mobile phase B was: $0 \rightarrow 15 \% \rightarrow 25 \% \rightarrow 25 \% \rightarrow 0$. The eluent flowed at $1 \mathrm{~mL} / \mathrm{min}$ and $25{ }^{\circ} \mathrm{C}$ and the UV detection was carried out at a wavelength of $250 \mathrm{~nm}$.

\subsection{Fourier transform infrared spectroscopy (FT-IR) analysis of pectin samples}

Samples $(2 \mathrm{mg})$ were mixed with $\mathrm{KBr}(0.5 \mathrm{~g})$ and pressed into $\mathrm{KBr}$ pellets before measurement. FT-IR spectra were collected with an IR spectrometer (Nicolet 5700; Thermo Fisher Scientific, MA, USA), using the absorbance mode in the frequency range of $4000-400 \mathrm{~cm}^{-1}$ and a resolution of $4 \mathrm{~cm}^{-1}$.

\subsection{Nuclear magnetic resonance (NMR) analysis of pectin samples}

Pectin samples were dispersed in $\mathrm{D}_{2} \mathrm{O}$ at $60{ }^{\circ} \mathrm{C}$ and lyophilized twice to obtain suspensions with the ultimate concentrations of 3-5\% (samples were dissolved as much as possible in order to achieve stronger signals in the spectra). NMR spectra of samples were measured by a $600 \mathrm{MHz}$ NMR spectrometer (DD2-600; Agilent Technologies Inc., CA, USA) at $25{ }^{\circ} \mathrm{C}$. The spectra were analyzed and processed using the MestReNova 6.1.1 (MestreLab Research, Santiago de Compostela, Spain)

\subsection{Atomic force microscope (AFM) analysis of pectin samples}

Pectin samples were dissolved in water with a concentration of $10 \mu \mathrm{g} / \mathrm{mL}$ and vibrated at the room temperature for $72 \mathrm{~h}$. Ten microliters of the dispersed pectin samples were dropped onto a mica splitting and dried in the air. Then the mica splitting was fixed to an AFM specimen disc. Nanostructures of three samples were observed using the AFM (AFM-D5A; MicroNano Corp., Shanghai, China) with a $\mathrm{Si}_{3} \mathrm{~N}_{4}$ tip (at a resonance frequency of $30 \mathrm{~Hz}$ and a scan rate of $0.5-2 \mathrm{~Hz}$ ). The 
collected images were analyzed and processed using the ChinaSPM 3.4.4 (MicroNano Corp., Shanghai, China).

\subsection{Statistical analysis}

All experiments above were repeated three times and the data was recorded as a mean \pm standard deviation. Analysis of experimental data was conducted by ANOVA $(p<0.05)$ and Duncan's multiple range tests using the SPSS 17.0 (SPSS Inc., Chicago, IL, USA). The figures were processed by Origin Software 8.5 (OriginLab Corp., MA, USA).

\section{Results and discussions}

3.1. Effects of ultrasound conditions on the $M_{w}$ and its polydispersity of pectin hydrolysates

\subsubsection{Effect of ultrasound intensity on the $M_{w}$ and its polydispersity of pectin hydrolysates}

The molecular weight of pectin hydrolysates after sonoenzymolysis treatment at 0-9 $\mathrm{W} \mathrm{mL}^{-1}$ intensities for $10 \mathrm{~min}$ is demonstrated in Fig. 1(a). The $M_{w}$ of pectin hydrolysates decreased from 48 to $39 \mathrm{kDa}$ as the ultrasound intensity increased from 0 to $4.5 \mathrm{~W} \mathrm{~mL} \mathrm{~m}^{-1}$. Then, it gradually increased to $50 \mathrm{kDa}$ as the intensity further increased to $9 \mathrm{~W} \mathrm{~mL}^{-1}$. Low-intensity ultrasound accelerated the enzymatic degradation process and achieved maximum effect at $4.5 \mathrm{~W} \mathrm{~mL} \mathrm{~m}^{-1}$, which was attributed to the increased pectinase activity with ultrasound treatment (Ma et al., 2016). As the $M_{w}$ of the sonoenzymolysis products became higher than that of the enzymolysis products, an antagonistic effect between ultrasound and pectinase was 
observed when the intensity exceeded $8.1 \mathrm{~W} \mathrm{~mL}^{-1}$. Excessive free radicals and strong shear forces generated from intense ultrasound conditions could disrupt enzyme conformations and inactivated pectinase (Ma et al., 2015; Ma et al., 2016), which leads to the reduction in the degradation extent.
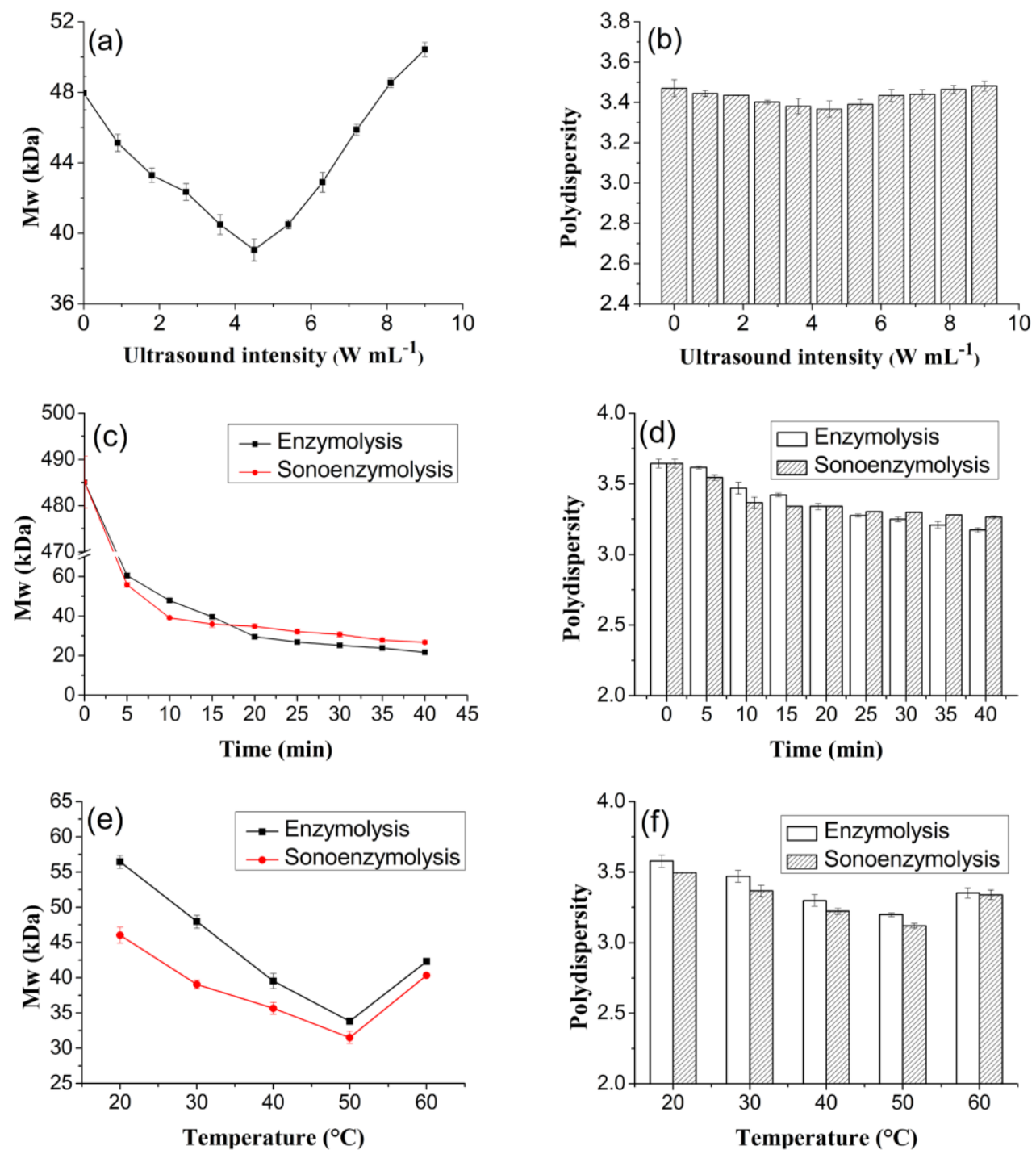

Fig. 1. Effects of operating conditions on the molecular weight and polydispersity of pectin hydrolysates. Effect of ultrasound intensity on the (a) molecular weight and (b) polydispersity of pectin hydrolysates; effect of ultrasound duration on the (c) molecular weight and (d) polydispersity of pectin hydrolysates; effect of temperature on the (e) molecular weight and (f) polydispersity of pectin hydrolysates.

Chain length of polymers is closely linked to their functions (Cooke \& Shi, 2006); thus, it is important to study the $M_{w}$ distributions of the degradation products. 
Fig. 1(b) shows the polydispersity index of pectin hydrolysates at each ultrasound intensity. The polydispersity decreased with the increase in ultrasound intensity until achieving its minimum value at $4.5 \mathrm{~W} \mathrm{~mL}^{-1}$, indicating a narrower $M_{w}$ distribution of pectin hydrolysates under ultrasound irradiation. As the low-intensity ultrasound improved pectinase activity and led to more complete hydrolysis, long chains of pectin were sheared, and the $M_{w}$ distributions became concentrated in the lower molecular weight regions. On the other hand, denaturation of pectinase under high-intensity $\left(>8.1 \mathrm{~W} \mathrm{~mL}^{-1}\right)$ ultrasonic fields resulted in decreased hydrolysis extent and higher polydispersity index as can be seen from Fig. 1(b).

\subsubsection{Effect of ultrasound duration on the $M_{w}$ and its polydispersity of pectin}

\section{hydrolysates}

The $M_{w}$ of pectin degraded by enzymolysis and sonoenzymolysis reactions (at $4.5 \mathrm{~W} \mathrm{~mL}^{-1}$ intensity) for $40 \mathrm{~min}$ is shown in Fig. 1(c). The $M_{w}$ was remarkably reduced (from $485 \mathrm{kDa}$ to $60 \mathrm{kDa}$ for enzymolysis pectin and $56 \mathrm{kDa}$ for sonoenzymolysis pectin, respectively) within 5 min for both reactions, indicating the high-efficiency of pectinase. Ultrasound irradiation reinforced the enzymolysis process and the maximum intensification was observed at $10 \mathrm{~min}$, when the $M_{w}$ decreased by $18.54 \%$ compared with that of the enzymolysis products. However, the enzymatic reactions were slowed down with a sonication time longer than $20 \mathrm{~min}$, suggesting that the pectinase was deactivated by prolonged ultrasound. On the other hand, $M_{w}$ degradation of both reactions flattened out after $20 \mathrm{~min}$; this can be attributed to the high methoxyl contents in pectin structures. The main component of 
pectinase used in the present work was polygalacturonase, which randomly cleaved glycosidic linkages next to free carboxyl groups (Sharma, Rathore \& Sharma, 2013). No de-esterification pretreatment was conducted prior to degradation reactions. The existence of methoxyl in HG regions handicapped further actions of pectinase; thus, the substrate would not be completely hydrolyzed under current conditions. Fig. 1(d) illustrates the corresponding variations of $M_{w}$ distributions for enzymolysis and sonoenzymolysis products. The polydispersity index of the original pectin was 3.64 , which was in accord with the reported polydispersity index for commercial pectin $(\mathrm{Li}$, Al-Assaf, Fang \& Phillips, 2013). The variation trends of polydispersity were similar to those of molecular weight. Short-time ultrasound treatments led to enhanced degradation extent and concentrated $M_{w}$ distributions of pectin hydrolysates, whereas prolonged ultrasound resulted in higher values of $M_{w}$ and polydispersity. Changes in pectinase activity under an ultrasonic field as mentioned above could explain these phenomena.

Results above showed that the pectin hydrolysis was enhanced with ultrasound at low intensity and short time, but it was retarded by the intense ultrasound conditions. The same tendency was observed for the sonoenzymolysis reactions of dextran (Bashari et al., 2013), xylan (Sun et al., 2015) and guar (Prajapat et al., 2016a). Sun et al. (Sun et al., 2015) studied the sono-enzymatic hydrolysis of xylan. Results demonstrated that productions of both reducing sugar and total soluble sugar were increased at an ultrasound power of $200 \mathrm{~W}$ and a time of $10 \mathrm{~min}$, as compared with the mere enzymatic process. However, the yield of reducing sugar decreased when the 
ultrasound intensity was over $200 \mathrm{~W}$ or the treatment time exceeded $10 \mathrm{~min}$,

\subsubsection{Effect of temperature on the $M_{w}$ and its polydispersity of pectin hydrolysates}

Fig. 1(e) and (f) represent the $M_{w}$ and the polydispersity index of enzymolysis and sonoenzymolysis products at a temperature range of $20{ }^{\circ} \mathrm{C}$ to $60{ }^{\circ} \mathrm{C}$ (at $10{ }^{\circ} \mathrm{C}$ interval), respectively. The $M_{w}$ and polydispersity of pectin hydrolysates first decreased and then increased with an increase in temperature. For both enzymolysis and sonoenzymolysis reactions, the maximum degradation extent was observed at $50{ }^{\circ} \mathrm{C}$, with the lowest $M_{w}$ and the narrowest distributions obtained. This suggested that the optimum temperature for pectinase with or without ultrasound treatment was both at $50{ }^{\circ} \mathrm{C}$. The lower degradation extent (expressed as the increased $M_{w}$ and polydispersity) at $60{ }^{\circ} \mathrm{C}$ was ascribed to the thermal inactivation of pectinase and decreased cavitational activity of ultrasound at high temperatures (Ma et al., 2016). On the other hand, $M_{w}$ and polydispersity index of the sonoenzymolysis products were lower than those of the enzymolysis products at each tested temperature, suggesting an improved degradation process under ultrasound irradiation.

\subsection{Synergistic effects of ultrasound and pectinase on degradation kinetics of} pectin $M_{w}$

Degradation kinetics of pectin $M_{w}$ for the sonolysis, enzymolysis and sonoenzymolysis reactions were studied with the zero-order, first-order and second-order kinetic models respectively and the correlation coefficients are listed in Table A.1. The second-order kinetic model was the most adequate for the degradation 
processes with all correlation coefficients higher than 0.97. Zhang et al. (Zhang et al., 2013a) studied the ultrasonic degradation of apple pectin at 5 to $45^{\circ} \mathrm{C}$. The reactions were fitted to $1 / M w_{t}-1 / M w_{0}=k t$, which was in conformity with this study. The second-order kinetic model was supposed to be suitable for a truly random degradation process (Roubroeks et al., 2001; Tommeraas \& Melander, 2008). Roubroeks et al. (Roubroeks et al., 2001) studied the enzymatic hydrolysis kinetics of oat $\beta$-glucan. The degradation rate was found to decrease as the reaction time increased, which was due to the different affinity of the enzyme for the different parts of the substrate. In this study, pectinase could randomly hydrolyze the $\alpha-1$, 4-glycosidic bond of pectin; thus the degradation kinetics followed the second-order kinetics model.

Fig. 2 depicts plots of $1 / M w_{t}-1 / M w_{0}$ against time for pectin depolymerized by sonolysis, enzymolysis and sonoenzymolysis reactions at a temperature range of $20{ }^{\circ} \mathrm{C}$ to $60{ }^{\circ} \mathrm{C}$. Rate constants for degradation reactions and values of synergistic coefficients were summarized in Table 1. Because of the low ultrasound intensity, rate constants of sonolysis reactions were remarkably lower than those of enzymolysis or sonoenzymolysis reactions. In sonolysis reactions, the $k$ value at $20{ }^{\circ} \mathrm{C}$ was higher than that at $30{ }^{\circ} \mathrm{C}$ (Fig. 2 (a)) due to the higher cavitational activity (Zhang et al., 2013a). However, $k$ became positively correlated with temperatures when the temperature was over $40{ }^{\circ} \mathrm{C}$, indicating that thermal degradation of pectin happened. For the pectinase-catalyzed degradation reactions with or without ultrasound treatment (Fig. 2 (b) and (c)), $k$ elevated as the temperature increased from $20^{\circ} \mathrm{C}$ to 
$50{ }^{\circ} \mathrm{C}$. Then it significantly reduced as the temperature further increased to $60{ }^{\circ} \mathrm{C}$, which was on account of the thermal inactivation of pectinase.
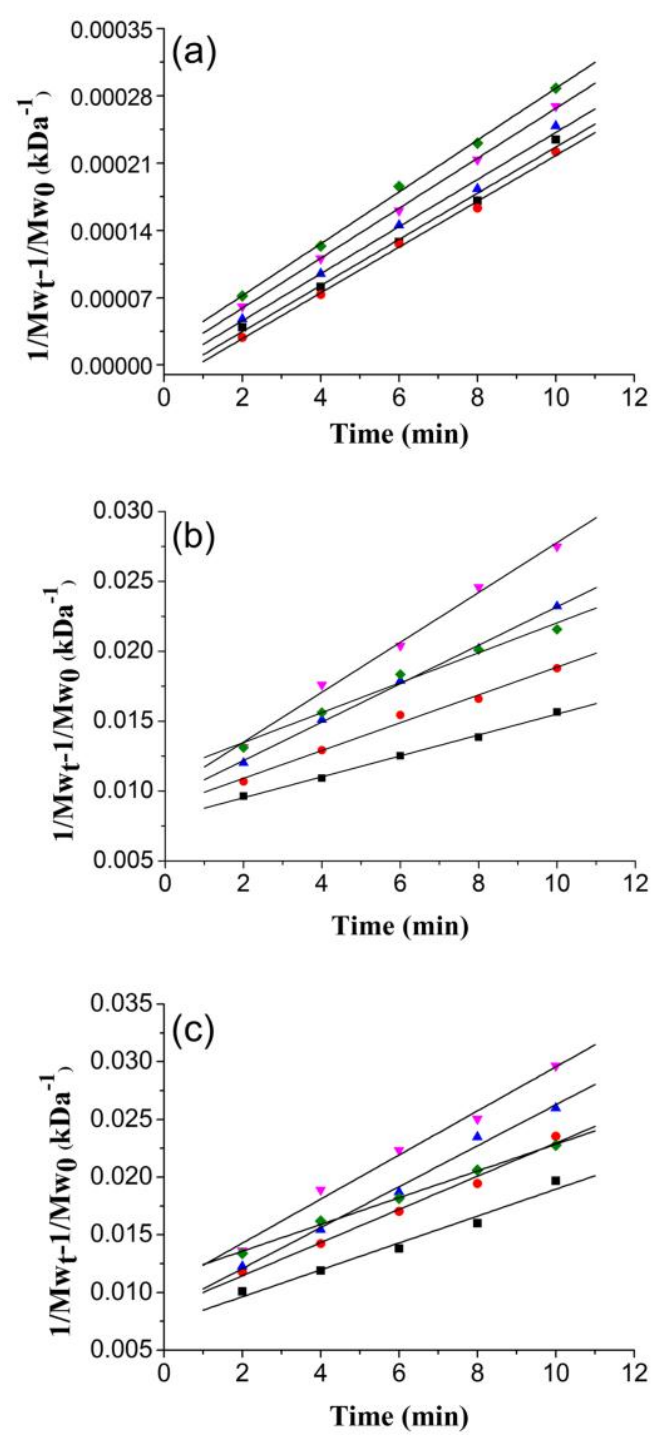

Fig. 2. The second-order degradation kinetics curves of pectin $M_{w}$ under (a) sonolysis, (b) enzymolysis and (c) sonoenzymolysis reactions. $20^{\circ} \mathrm{C} \bullet 30^{\circ} \mathrm{C} \boldsymbol{\Delta} 40^{\circ} \mathrm{C} \boldsymbol{\nabla} 50^{\circ} \mathrm{C} \bullet 60^{\circ} \mathrm{C}$ 


\section{Table 1}

Rate constants of different degradation reactions and synergistic coefficients at the temperature range of $20-60{ }^{\circ} \mathrm{C}($ mean $\pm \mathrm{SD})$.

\begin{tabular}{lllllll}
\hline Parameters & Processing & 20 & 30 & 40 & 50 & 60 \\
\hline & $\begin{array}{l}\text { Sonolysis } \\
\left(\times 10^{-8}\right)\end{array}$ & $2.40 \pm 0.11$ & $2.38 \pm 0.08$ & $2.45 \pm 0.11$ & $2.60 \pm 0.03$ & $2.70 \pm 0.06$ \\
& $\begin{array}{l}\text { Enzymolysis } \\
\left(\times 10^{-6}\right)\end{array}$ & $0.75 \pm 0.00$ & $0.99 \pm 0.01$ & $1.37 \pm 0.00$ & $1.78 \pm 0.01$ & $1.07 \pm 0.01$ \\
$\left(\mathrm{~mol} \mathrm{~g}^{-1} \mathrm{~min}^{-1}\right)$ & & & & & \\
& $\begin{array}{l}\text { Sonoenzymolysis } \\
\left(\times 10^{-6}\right)\end{array}$ & $1.16 \pm 0.01$ & $1.44 \pm 0.01$ & $1.77 \pm 0.01$ & $1.91 \pm 0.01$ & $1.16 \pm 0.00$ \\
\hline \multirow{3}{*}{$R^{2}$} & Sonolysis & 0.9913 & 0.9950 & 0.9920 & 0.9993 & 0.9977 \\
& $\begin{array}{l}\text { Enzymolysis } \\
\text { Sonoenzymolysis }\end{array}$ & 0.9959 & 0.9851 & 0.9971 & 0.9927 & 0.9787 \\
\hline Synergy (\%) & & $(+) 33.45$ & $(+) 29.31$ & $(+) 21.21$ & $(+) 5.45$ & $(+) 5.43$ \\
Synergistic effect & & Synergistic & Synergistic & Synergistic & Synergistic & Synergistic \\
\hline
\end{tabular}

On the other hand, positive values of synergistic coefficients were observed within the temperature range of $20-60{ }^{\circ} \mathrm{C}$ (Table 1), demonstrating that a greater degradation degree was obtained under the simultaneous function of ultrasound and pectinase. In addition to the elevated enzyme activity, dispersed substrate structures and a more homogeneous system acquired under an ultrasonic field also contributed to this synergistic effect. The synergistic coefficient significantly decreased with the increase in temperatures, which was supposed to be attributed to the severe deformation of pectinase structures (Ma et al., 2015; Ma et al., 2016) and the lower cavitational activity at high temperatures. In conclusion, the synergistic effects of ultrasound and pectinase in pectin $M_{w}$ degradation were stronger at temperatures below $40{ }^{\circ} \mathrm{C}$.

\subsection{DM and DAc of pectin samples}

Table 2 indicates the DM and DAc of the original pectin, enzymolysis pectin and sonoenzymolysis pectin; and the corresponding HPLC chromatograms are shown in 
Fig. A. 1. There was no significant difference in DM values of the enzymolysis products and the control, since the pectinase preparation did not contain pectin methyl esterase. Ultrasonic shear forces could mechanically cleave the C-O bond of ester linkages; meanwhile, free radicals generated from cavitation could react with the methyl ester bond to produce $\cdot \mathrm{OCH}_{3}$, which led to the breakage of the $\mathrm{C}-\mathrm{O}$ bond (Zhang et al., 2013b). In a previous study (Zhang et al., 2013b), ultrasound was reported to decrease the $\mathrm{DM}$ of citrus pectin by $5 \%$ at $302 \mathrm{~W} \mathrm{~cm}^{-2}$ intensity and $0{ }^{\circ} \mathrm{C}$ for $30 \mathrm{~min}$. With coinstantaneous action of pectinase, this study utilized milder ultrasound conditions and obtained a better demethoxylation effect. As can be seen from Table 2, DM was significantly decreased from $54.63 \%$ to $42.27 \%$ with the sonoenzymolysis treatment compared to the control. A coordination mechanism of ultrasound and pectinase was proposed to explain this phenomenon. In sonoenzymolysis reactions, pectin substrates were rapidly split into smaller segments by pectinase hydrolysis, which increased the exposure of methoxyl groups for ultrasonic attack; the subsequent release of non-esterified substrates would be favored by pectinase and resulted in a more complete degradation. Therefore, cooperation of ultrasound and pectinase might be another mechanism for their synergistic effects.

\section{Table 2}

The DM and DAc of pectin with different treatments (mean $\pm \mathrm{SD}$ ).

\begin{tabular}{lll}
\hline Samples & DM (\%) & DAc (\%) \\
\hline Control & $54.63 \pm 1.01^{a}$ & $1.56 \pm 0.17^{a}$ \\
Enzymolysis & $53.35 \pm 1.15^{a}$ & $1.59 \pm 0.13^{a}$ \\
Sonoenzymolysis & $42.27 \pm 0.87^{b}$ & $1.52 \pm 0.13^{a}$ \\
\hline
\end{tabular}

Note: values with different italic superscript letters $(a-b)$ in the same column within each pectin indicate significant differences as estimated by Duncan's multiple range test $(\mathrm{P}<0.05)$. 
However, there were no significant variations in DAc of pectin hydrolysates compared to the control, demonstrating that both ultrasound and pectinase could maintain the acetyl group in pectin structures. This phenomenon was consistent with a previous study on the DAc of chitosan with the ultrasound treatment (Lima \& Andrade, 2010). Results indicated that the combination of ultrasound and pectinase could significantly decrease the DM of pectin while still maintaining its DAc.

\subsection{Monosaccharide composition of pectin samples}

The monosaccharide components of three pectin samples are expressed in mol\% and listed in Table 3 (the HPLC chromatograms are shown in Fig. A. 2). Since Glc was supposed to be an impurity from the extraction process (Zhang et al., 2013a), the pectin substrate mainly consisted of six monosaccharides, including GalUA, Gal, Rha, Ara, Xyl and Fuc. Amongst these, GalUA was from HG and RG-I main chains; Rha was from RG-I main chain; Gal and Ara were from RG-I side chains; Fuc and Xyl were from side chains of RG-II and XG regions, respectively. Meanwhile, ratios of Rha/GalUA and (Gal + Ara)/Rha were calculated to be the indicators of RG-I main chains and side chains contents. According to Table 3, the main conclusions were summarized as follows: (1) the GalUA content was predominant in pectin, but significantly reduced in the enzymolysis and sonoenzymolysis products (a larger decrease was achieved in the sonoenzymolysis products); (2) contents of Rha and Ara were significantly increased in the sonoenzymolysis products compared to the control and the enzymolysis pectin; (3) the ratio of Rha/GalUA was significantly increased whereas $(\mathrm{Gal}+\mathrm{Ara}) / \mathrm{Rha}$ was unchanged in the sonoenzymolysis products compared 
with the other two samples. Results suggested a notable increase in RG-I contents of degraded products. More remarkable changes were observed in the sonoenzymolysis pectin when compared to the enzymolysis pectin, due to the more severe hydrolysis of pectin's main chain under an ultrasonic field. This phenomenon was ascribed to two reasons: firstly, ultrasound could elevate pectinase activity and lead to the increased hydrolysis rate; secondly, as the polygalacturonase needed non-esterified substrates, the removal of methoxyl groups under ultrasound irradiation provided more action sites for pectinase, which further increased the degradation extent of the pectin main chain. Results indicated that the decomposition of the HG regions was the main reason for the decreased $M_{w}$ in the sonoenzymolysis reactions.

\section{Table 3}

Monosaccharide component (mol\%) of pectin with different treatments (mean $\pm \mathrm{SD}$ ).

\begin{tabular}{llll}
\hline Monosaccharides & Control & $\begin{array}{l}\text { Enzymolysis } \\
\text { products }\end{array}$ & $\begin{array}{l}\text { Sonoenzymolysis } \\
\text { products }\end{array}$ \\
\hline Rha & $8.85 \pm 0.06^{b}$ & $10.10 \pm 0.32^{b}$ & $11.89 \pm 0.76^{a}$ \\
GalA & $68.19 \pm 0.31^{a}$ & $63.93 \pm 0.58^{b}$ & $59.26 \pm 1.19^{c}$ \\
Glu & $4.34 \pm 0.06^{a}$ & $4.78 \pm 0.40^{a}$ & $5.95 \pm 0.76^{a}$ \\
Gal & $12.44 \pm 0.08^{a}$ & $15.04 \pm 0.10^{a}$ & $14.77 \pm 0.45^{a}$ \\
Xyl & $1.38 \pm 0.05^{a}$ & $1.28 \pm 0.09^{a}$ & $1.51 \pm 0.04^{a}$ \\
Ara & $3.30 \pm 0.04^{b}$ & $3.38 \pm 0.25^{b}$ & $4.16 \pm 0.24^{a}$ \\
Fuc & $1.50 \pm 0.09^{a}$ & $1.48 \pm 0.47^{a}$ & $2.45 \pm 0.16^{a}$ \\
Rha/ GalA & $0.13 \pm 0.00^{b}$ & $0.16 \pm 0.01^{b}$ & $0.20 \pm 0.02^{a}$ \\
(Gal+Ara)/Rha & $1.78 \pm 0.02^{a}$ & $1.82 \pm 0.02^{a}$ & $1.61 \pm 0.19^{a}$ \\
\hline
\end{tabular}

Note: Rha: GalA and $(\mathrm{Gal}+\mathrm{Ara})$ : Rha represent molar ratios; values with different italic superscript letters $(a-c)$ in the same column within each pectin indicate significant differences as estimated by Duncan's multiple range test $(\mathrm{P}<0.05)$.

High-intensity ultrasound was previously reported to induce degradation of RG-I side chains rather than main chains of pectin (Zhang et al., 2013a), which was adverse to the bioactivity retention of pectin. The negative influence of ultrasound was eliminated in the present work given the low intensity of the applied ultrasound 
conditions. Synergistic action of ultrasound and pectinase was thus proven to be a more ideal method for MP preparation.

\subsection{FT-IR analysis of pectin samples}

Fig. 3 illustrates the infrared spectra of pectin with and without degradation treatments. An absorption at the wavelength range of 3000 to $3700 \mathrm{~cm}^{-1}$ was contributed by the stretching vibration of $\mathrm{O}-\mathrm{H}$. The absorption peak was broadened due to the formation of intra- and intermolecular hydrogen bonds. A weak absorption at $2933 \mathrm{~cm}^{-1}$ was derived from $\mathrm{C}-\mathrm{H}$ stretching, including $\mathrm{CH}, \mathrm{CH}_{2}$, and $\mathrm{CH}_{3}$ stretching and bending vibrations (Gnanasambandam \& Proctor, 2000). The methylesterified carbonyl groups $(\mathrm{C}=\mathrm{O})$ and the ionic carboxyl groups $(\mathrm{COO}-)$ occurred at $1743 \mathrm{~cm}^{-1}$ and $1622 \mathrm{~cm}^{-1}$, respectively. The ratio of the peak area at $1743 \mathrm{~cm}^{-1}$ over the sum of the area at $1743 \mathrm{~cm}^{-1}$ and $1622 \mathrm{~cm}^{-1}$ determined the DM of pectin samples (Zhang et al., 2013a). Three absorption peaks at regions of $1000-1200 \mathrm{~cm}^{-1}$ indicated the pyranose configuration of pectin. Strong bands occurring at $1103 \mathrm{~cm}^{-1}$ and $1016 \mathrm{~cm}^{-1}$ represented high contents of uronic acid in the samples and were the characteristic absorptions of pectin (Coimbra, Barros, Barros, Rutledge \& Delgadillo, 1998).

As can be seen from Fig. 3, the pyranose configuration of pectin samples was unchanged with enzymolysis and sonoenzymolysis treatments. The DM of pectin did not change after pectinase-catalyzed hydrolysis; instead, it was significantly reduced under an ultrasonic field, which was in line with the analysis of HPLC. 

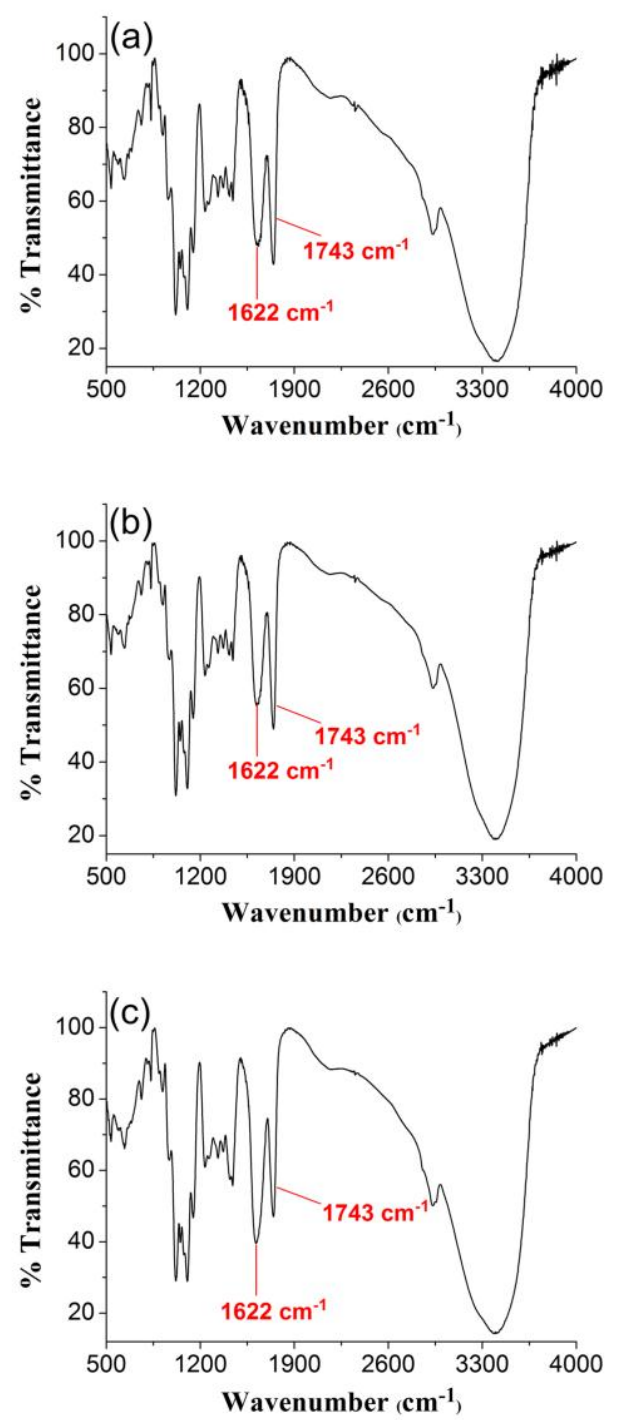

Fig. 3. The IR spectra of (a) original pectin, (b) enzymolysis pectin and (c) sonoenzymolysis pectin.

\subsection{NMR analysis of pectin samples}

Given differences in the $M_{w}$ and solubility of three samples, the NMR measurement was conducted with different sample concentrations (sonoenzymolysis pectin $>$ enzymolysis pectin $>$ original pectin), and thereby the peak heights in the spectra were incomparable. Results of NMR spectra were thus used for the analysis of configurations and glycosidic bonds of samples. Fig. A. 3 shows the ${ }^{1} \mathrm{H}$ NMR spectra of pectin with and without degradation treatments, and the chemical shifts of major signals are listed in the Table A. 2. There were no detected signals nearby 5.4 ppm, 
which confirmed the pyranose configuration of the samples. Signals at $5.09 \mathrm{ppm}$ for untreated pectin and at 5.03 ppm for degradation products denoted H-1 absorption of free GalUA units, while signals at $5.03 \mathrm{ppm}$ for untreated pectin and $4.99 \mathrm{ppm}$ for degradation products represented H-1 absorption of the esterified units (Winning, Viereck, Norgaard, Larsen \& Engelsen, 2007). The chemical shifts of H-1 were all beyond $4.95 \mathrm{ppm}$ for three samples, demonstrating that the $\alpha$-glycoside linkage of GalUA was unaltered during the enzymolysis and sonoenzymolysis reactions. Signals at $3.75 \mathrm{ppm}$ referred to the $-\mathrm{CH}_{3}$ protons binding to the GalUA ester groups (Patra, Das, Behera, Maiti \& Islam, 2012). Peaks at $2.12 \mathrm{ppm}$ and $2.01 \mathrm{ppm}$ were attributed to acetyl groups attached to 2- and 3-O-GalA, respectively (Majoros, Keszler, Woehler, Bull \& Baker, 2003; Tamaki, Konishi, Fukuta \& Tako, 2008). Results showed that acetyl groups were mainly linked to 3-O-GalA (Fig. 4). Peaks at 1.20 and $1.26 \mathrm{ppm}$ were assigned to $-\mathrm{CH}_{3}$ protons of L-Rha bound at $\mathrm{O}-2$ and $\mathrm{O}-2,4$ sites, respectively. In conclusion, there were no significant changes in ${ }^{1} \mathrm{H}$ NMR spectra for the three samples except for slight shifts of signals.

Fig. A. 4 demonstrates the ${ }^{13} \mathrm{C}$ NMR spectra of pectin samples. A stronger signal-to-noise ratio was observed for the enzymolysis and sonoenzymolysis samples as compared to the control, given the lower $M_{w}$ and higher solubility of the degraded pectin. Six major signals in the spectra were derived from D-GalUA (Tamaki et al., 2008), indicating that the pectin main chain did not alter under both degradation reactions. This was in line with the results of the monosaccharide component analysis. Signals at $55.37 \mathrm{ppm}$ referred to $-\mathrm{CH}_{3}$ carbon binding to the GalUA carboxyl groups. 
Peaks occurring at 173 and 177 ppm were derived from carboxyl groups with or without esterification, respectively. GalUA residues in the three samples were partly methoxylated according to the results. Conclusions were in agreement with results of HPLC and IR: functions of ultrasound and pectinase did not change the conformations and bond linkages of pectin; the primary structure of pectin was maintained during the degradation processes.

\subsection{AFM analysis of pectin samples}

Fig. 4 shows the AFM images of pectin molecules before and after the degradation treatments. Four main nanostructures including the linear strands (ls), single branch (br), multiple branches (mbr) and polymer (p) were marked in the images. Looking at Fig. 4 (a), various branches were attached to the linear main chain of pectin and gave a tangled, complex polymeric structure. The chain width and chain height measured for the original pectin were concentrated in the ranges of 70-90 nm and $0.5-0.9 \mathrm{~nm}$, respectively, which were close to the reported ranges for pectin's chain width and chain height (Round, Rigby, MacDougall \& Morris, 2010; Zhang et al., 2013b). However, when pectin molecules were hydrolyzed by pectinase (Fig. 4 (b)), the polymer structures disappeared and the long chains were cleaved into shorter ones; when ultrasound was introduced during the enzymatic reactions (Fig. 4 (c)), the multiple branches were further degraded and more short strands appeared. The chain width and chain height for the enzymolysis and sonoenzymolysis pectin samples decreased to the ranges of $20-40 \mathrm{~nm}$ and $0.1-0.5 \mathrm{~nm}$, respectively, indicating that the tangled structures were unfolded during the degradation reactions. Ultrasound was 
observed to promote the enzymatic process and the polymeric structures of pectin were effectively sheared into smaller and simpler segments under the sonochemical-enzymatic effects. Also, the increased amounts of short chains in pectin structures could be an explanation for the lower $M_{w}$ and its more concentrated distributions as mentioned before.

(a)

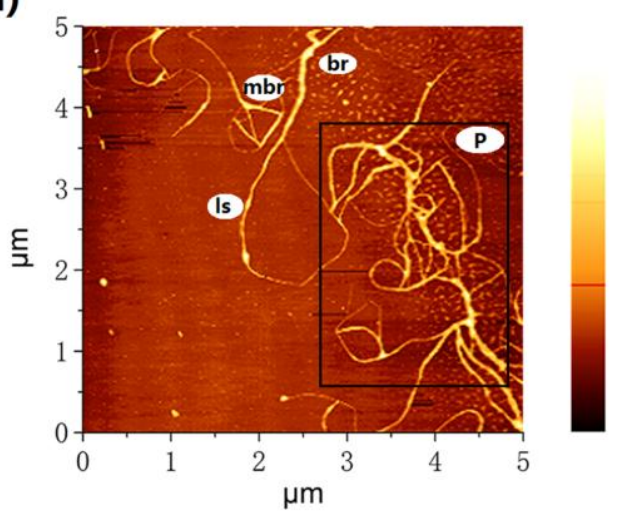

(b)

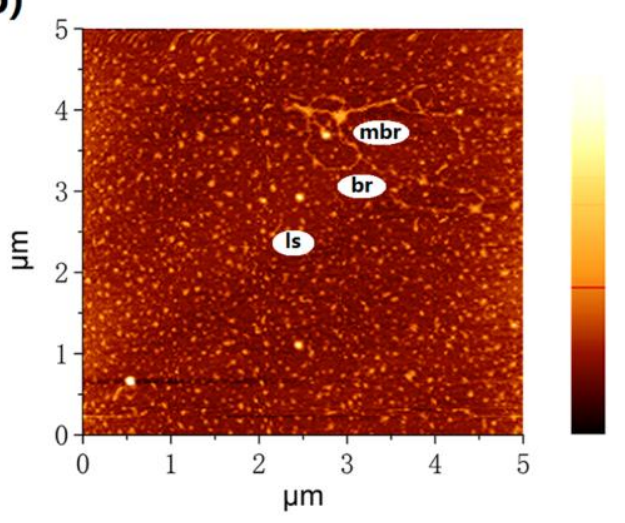

(c)

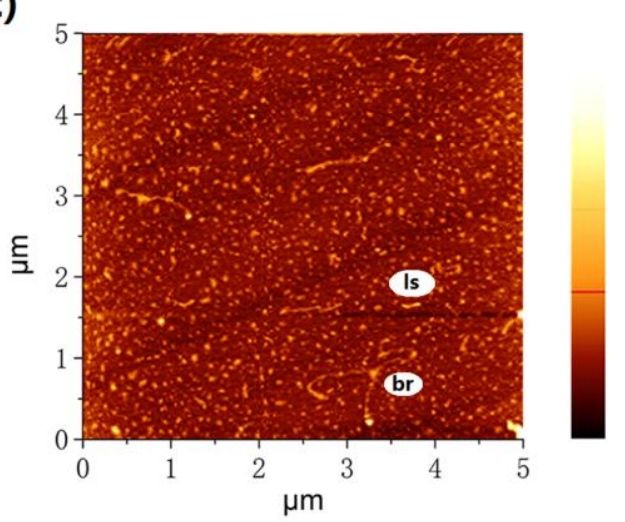

Fig. 4. The AFM images of (a) original pectin, (b) enzymolysis pectin and (c) sonoenzymolysis pectin (with the scan area of $5 \mu \mathrm{m} \times 5 \mu \mathrm{m}$ ). 
In a study by Zhang et al. (Zhang et al., 2015), pectin was degraded under an ultrasonic field at $181 \mathrm{~W} \mathrm{~cm}^{-1}$ intensity for $15 \mathrm{~h}$ at $25^{\circ} \mathrm{C}$; the branch structures disappeared after ultrasound treatment. However, in this study, braches still existed after ultrasound. This phenomenon together with the monosaccharide analysis suggested that cooperation of mild ultrasound and pectinase mainly induced degradation in the pectin main chains, while the RG-I regions were stable under the current sonoenzymolysis conditions.

\section{Conclusions}

The current study detected the dynamic variations in pectin $M_{w}$ and its distributions under sono-enzymatic conditions. Degradation of pectin $M_{w}$ was proven to be a random process as ascribed by the kinetics model $1 / M w_{t}-1 / M w_{0}=k t$. The synergistic effects of ultrasound and pectinase were observed at the temperature range of $20{ }^{\circ} \mathrm{C}$ to $60{ }^{\circ} \mathrm{C}$, and were more obvious below $40{ }^{\circ} \mathrm{C}$. On the other hand, combination of ultrasound and pectinase significantly decreased the DM of pectin but still maintained its DAc. The sonoenzymolysis process could protect the RG-I regions of the pectin sample, while it can cause severe degradation in the pectin $\mathrm{HG}$ regions. Based on these conclusions, the possible degradation paths of pectin under enzymolysis and sonoenzymolysis reactions are summarized in Fig. 5. Results of this research work suggested an alternative method for MP processing. 


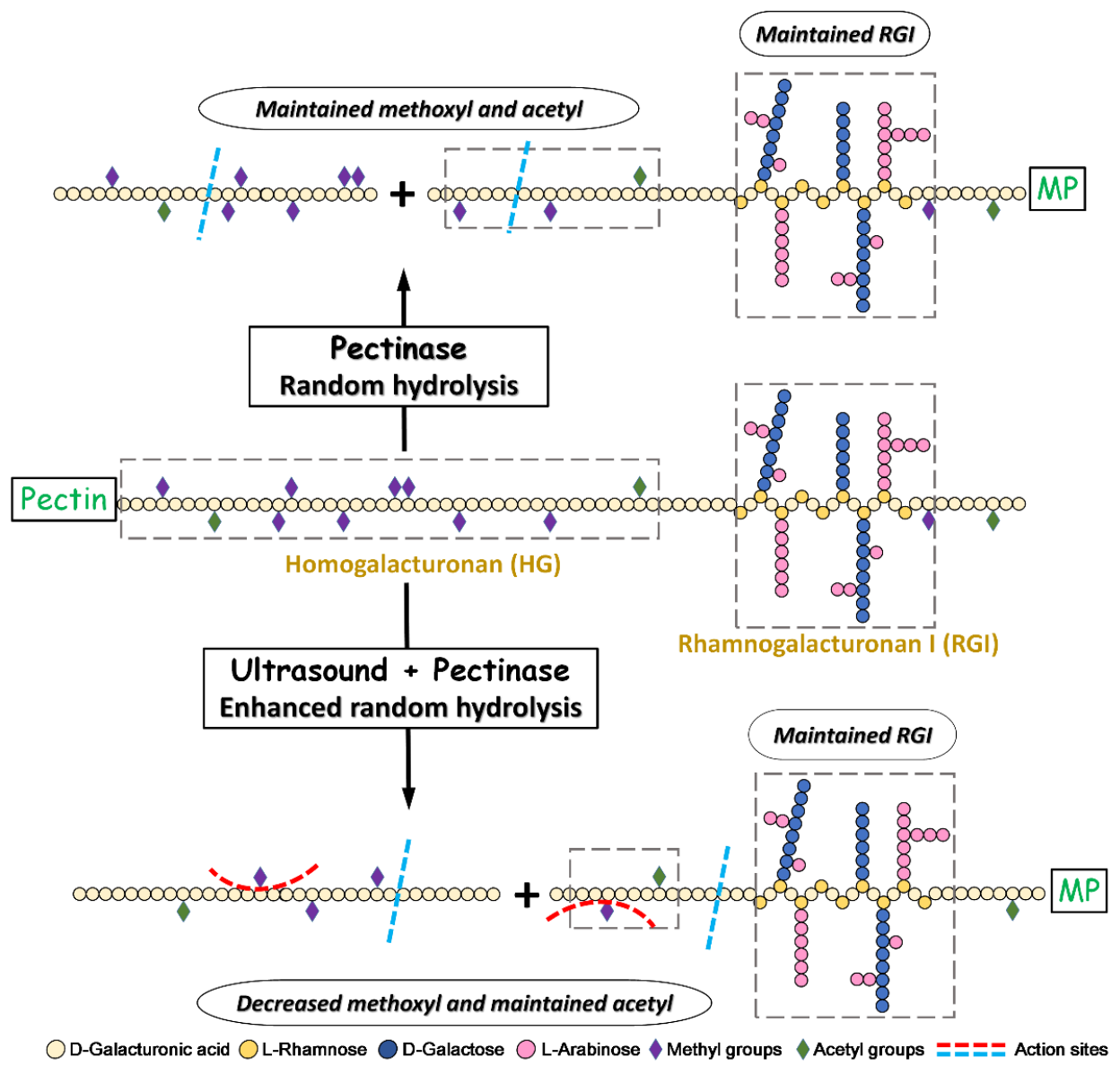

Fig. 5. The schematic diagram of pectin degradation path under enzymolysis and sonoenzymolysis reactions.

\section{Appendices}

Table A.1; Table A.2; Fig. A. 1; Fig. A. 2

\section{Acknowledgement}

This work was financially supported by National Natural Science Foundation of

China (Project 31371872).

\section{References}

Bashari, M., Eibaid, A., Wang, J., Tian, Y., Xu, X., \& Jin, Z. (2013). Influence of low ultrasound intensity on the degradation of dextran catalyzed by dextranase. Ultrasonics Sonochemistry, 20(1), 155-161.

Coimbra, M. A., Barros, A., Barros, M., Rutledge, D. N., \& Delgadillo, I. (1998). 
Multivariate analysis of uronic acid and neutral sugars in whole pectic samples by FT-IR spectroscopy. Carbohydrate Polymers, 37(3), 241-248.

Cooke, D. M., \& Shi, A. (2006). Effects of polydispersity on phase behavior of diblock copolymers. Macromolecules, 39(19), 6661-6671.

Fan, Y., Cheng, H., Liu, D., Zhang, X., Wang, B., Sun, L., Tai, G., \& Zhou, Y. (2010). The Inhibitory Effect of Ginseng Pectin on L-929 Cell Migration. Archives of Pharmacal Research, 33(5), 681-689.

Gnanasambandam, R., \& Proctor, A. (2000). Determination of pectin degree of esterification by diffuse reflectance Fourier transform infrared spectroscopy. Food Chemistry, 68(3), 327-332.

Gunning, A. P., Bongaerts, R. J. M., \& Morris, V. J. (2009). Recognition of galactan components of pectin by galectin-3. FASEB Journal, 23(2), 415-424.

Islam, M. N., Zhang, M., \& Adhikari, B. (2014). The Inactivation of Enzymes by Ultrasound-A Review of Potential Mechanisms. Food Reviews International, 30(1), $1-21$.

Levigne, S., Thomas, M., Ralet, M. C., Quemener, B., \& Thibault, J. F. (2002). Determination of the degrees of methylation and acetylation of pectins using a C18 column and internal standards. Food Hydrocolloids, 16(6), 547-550.

Li, X., Al-Assaf, S., Fang, Y., \& Phillips, G. O. (2013). Characterisation of commercial LM-pectin in aqueous solution. Carbohydrate Polymers, 92(2), $1133-1142$.

Lima, F. F., \& Andrade, C. T. (2010). Effect of melt-processing and ultrasonic 
treatment on physical properties of high-amylose maize starch. Ultrasonics Sonochemistry, 17(4), 637-641.

Lofgren, C., Guillotin, S., \& Hermansson, A. M. (2006). Microstructure and kinetic rheological behavior of amidated and nonamidated LM pectin gels. Biomacromolecules, 7(1), 114-121.

Ma, H., Huang, L., Jia, J., He, R., Luo, L., \& Zhu, W. (2011). Effect of energy-gathered ultrasound on Alcalase. Ultrasonics Sonochemistry, 18(1), 419-424.

Ma, X., Wang, W., Zou, M., Ding, T., Ye, X., \& Liu, D. (2015). Properties and Structures of Commercial Polygalacturonase with Ultrasound Treatment: Role of Ultrasound in Enzyme Activation. RSC Advances, 5, 107591-107600.

Ma, X., Zhang, L., Wang, W., Zou, M., Ding, T., Ye, X., \& Liu, D. (2016). Synergistic Effect and Mechanisms of Combining Ultrasound and Pectinase on Pectin Hydrolysis. Food and Bioprocess Technology, 9(7), 1249-1257.

Majoros, I. J., Keszler, B., Woehler, S., Bull, T., \& Baker, J. R. (2003). Acetylation of poly(amidoamine) dendrimers. Macromolecules, 36(15), 5526-5529.

Maxwell, E. G., Belshaw, N. J., Waldron, K. W., \& Morris, V. J. (2012). Pectin - An emerging new bioactive food polysaccharide. Trends in Food Science \& Technology, 24(2), 64-73.

MAY, C. D. (1990). INDUSTRIAL PECTINS - SOURCES, PRODUCTION AND APPLICATIONS. Carbohydrate Polymers, 12(1), 79-99.

Patra, P., Das, D., Behera, B., Maiti, T. K., \& Islam, S. S. (2012). Structure elucidation of an immunoenhancing pectic polysaccharide isolated from aqueous 
extract of pods of green bean (Phaseolus vulgaris L.). Carbohydrate Polymers, 87(3), 2169-2175.

Prajapat, A. L., Subhedar, P. B., \& Gogate, P. R. (2016a). Ultrasound assisted enzymatic depolymerization of aqueous guar gum solution. Ultrasonics Sonochemistry, 29, 84-92.

Prajapat, A. L., Das, P., \& Gogate, P. R. (2016b). A novel approach for intensification of enzymatic depolymerization of carboxymethyl cellulose using ultrasonic and ultraviolet irradiations. Chemical Engineering Journal, 290, 391-399.

Roubroeks, J. P., Andersson, R., Mastromauro, D. I., Christensen, B. E., \& Aman, P. (2001). Molecular weight, structure and shape of oat $(1 \longrightarrow 3),(1 \longrightarrow 4)-$ beta-D-glucan fractions obtained by enzymatic degradation with $(1 \longrightarrow$ 4)-beta-D-glucan 4-glucanohydrolase from Trichoderma reesei. Carbohydrate Polymers, 46(3), $275-285$.

Round, A. N., Rigby, N. M., MacDougall, A. J., \& Morris, V. J. (2010). A new view of pectin structure revealed by acid hydrolysis and atomic force microscopy. Carbohydrate Research, 345(4), 487-497.

Sharma, N., Rathore, M., \& Sharma, M. (2013). Microbial pectinase: sources, characterization and applications. Reviews in Environmental Science and Bio-Technology, 12(1), 45-60.

Subhedar, P. B., \& Gogate, P. R. (2013). Intensification of Enzymatic Hydrolysis of Lignocellulose Using Ultrasound for Efficient Bioethanol Production: A Review. Industrial \& Engineering Chemistry Research, 52(34), 11816-11828. 
Subhedar, P. B., \& Gogate, P. R. (2014). Enhancing the activity of cellulase enzyme using ultrasonic irradiations. Journal of Molecular Catalysis B-Enzymatic, 101, $108-114$.

Sun, J., Zhang, Z., Xiao, F., \& Jin, X. (2015). Production of xylooligosaccharides from corncobs using ultrasound-assisted enzymatic hydrolysis. Food Science and Biotechnology, 24(6), 2077-2081.

Sun, Y., Ma, G., Ye, X., Kakuda, Y., \& Meng, R. (2010). Stability of all-trans-beta-carotene under ultrasound treatment in a model system: Effects of different factors, kinetics and newly formed compounds. Ultrasonics Sonochemistry, 17(4), 654-661.

Szabo, O. E., \& Csiszar, E. (2013). The effect of low-frequency ultrasound on the activity and efficiency of a commercial cellulase enzyme. Carbohydrate Polymers, 98(2), 1483-1489.

Tamaki, Y., Konishi, T., Fukuta, M., \& Tako, M. (2008). Isolation and structural characterisation of pectin from endocarp of Citrus depressa. Food Chemistry, 107(1), $352-361$.

Tommeraas, K., \& Melander, C. (2008). Kinetics of hyaluronan hydrolysis in acidic solution at various pH values. Biomacromolecules, 9(6), 1535-1540.

Warmoeskerken, M., van der Vlist, P., Moholkar, V. S., \& Nierstrasz, V. A. (2002). Laundry process intensification by ultrasound. Colloids and Surfaces A-Physicochemical and Engineering Aspects, 210(2-3), 277-285.

Winning, H., Viereck, N., Norgaard, L., Larsen, J., \& Engelsen, S. B. (2007). 
Quantification of the degree of blockiness in pectins using H-1 NMR spectroscopy and chemometrics. Food Hydrocolloids, 21(2), 256-266.

Zhang, L., Ye, X., Ding, T., Sun, X., Xu, Y., \& Liu, D. (2013a). Ultrasound effects on the degradation kinetics, structure and rheological properties of apple pectin. Ultrasonics Sonochemistry, 20(1), 222-231.

Zhang, L., Ye, X., Xue, S. J., Zhang, X., Liu, D., Meng, R., \& Chen, S. (2013b).

Effect of high-intensity ultrasound on the physicochemical properties and nanostructure of citrus pectin. Journal of the Science of Food and Agriculture, 93(8), 2028-2036.

Zhang, L., Zhang, X., Liu, D., Ding, T., \& Ye, X. (2015). Effect of degradation methods on the structural properties of citrus pectin. LWT-Food Science and Technology, 61(2), 630-637.

Zhang, Y., Fu, E., \& Liang, J. (2008). Effect of Ultrasonic Waves on the Saccharification Processes of Lignocellulose. Chemical Engineering \& Technology, 31(10), 1510-1515. 